\title{
Hormone levels and cognitive function in postmenopausal midlife women
}

\author{
Joanne Ryan, ${ }^{\text {a,b }}$ Frank Z. Stanczyk, ${ }^{\mathrm{c}}$ Lorraine Dennerstein, ${ }^{\mathrm{a}}$ Wendy J. Mack, ${ }^{\mathrm{d}}$ \\ Margaret S. Clark, ${ }^{\mathrm{a}}$ Cassandra Szoeke, ${ }^{\mathrm{a}, 1}$ Daniel Kildea, ${ }^{\mathrm{e}}$ Victor W. Henderson ${ }^{\mathrm{f}, *}$
}

${ }^{a}$ Department of Psychiatry and National Ageing Research Institute, The University of Melbourne 3010, Victoria, Australia

${ }^{\mathrm{b}}$ Inserm, U888, Montpellier, F-34093 France; Univ Montpellier1, Montpellier, F-34000 France ${ }^{\mathrm{c}}$ Department of Obstetrics and Gynecology, Keck School of Medicine, University of Southern California, Los Angeles, CA, 90033, USA

${ }^{\mathrm{d}}$ Department of Preventive Medicine, Keck School of Medicine, University of Southern California, Los Angeles, CA, 90033, USA

${ }^{\mathrm{e} S c h o o l ~ o f ~ M a t h e m a t i c a l ~ a n d ~ G e o s p a t i a l ~ S c i e n c e s, ~ R M I T ~ U n i v e r s i t y ~ o f ~ M e l b o u r n e, ~ M e l b o u r n e ~ 3000, ~}$ Australia

${ }^{\mathrm{f}}$ Departments of Health Research and Policy (Epidemiology) and of Neurology and Neurological Sciences, Stanford University, Stanford, CA, 94305, USA

*Corresponding author: Stanford University School of Medicine, 259 Campus Drive, Stanford, California 94305-5405, USA. Tel. 1-650-723-5456; Fax: 1-650-725-6951; Email: vhenderson@ stanford.edu ${ }^{1}$ Present address: CSIRO Preventative Health Flagship and the National Ageing Research Institute (NARI); The University of Melbourne, Parkville 3052, Victoria, Australia

Authors' email addresses: joanne.ryan@inserm.fr (J. Ryan), fstanczyk@socal.rr.com (F.Z. Stanczyk), lorrdenn@aol.com (L. Dennerstein),wmack@usc.edu (W.J. Mack), mclark@comcen.com.au (M.S. Clark), cassandra.szoeke@csiro.au (C. Szoeke), dan.kildea@optusnet.com.au (D. Kildea), vhenderson@ stanford.edu 
Ryan et al., 2

(V.W. Henderson) 


\begin{abstract}
Gonadal hormones may influence cognitive function. Postmenopausal midlife women in the populationbased Melbourne Women's Midlife Health Project cohort were administered a comprehensive battery of neuropsychological tests on two occasions two years apart. Participants ( $n=148$, mean age 60 years) had undergone natural menopause and were not using hormone therapy. Estrone, total and free estradiol, and total and free testosterone levels were measured at time of the first testing. Principal component analysis identified four cognitive factors. In multiple linear regression analyses, better semantic memory performance was associated with higher total $(\mathrm{p}=0.02)$ and free $(\mathrm{p}=0.03)$ estradiol levels and a lower ratio of testosterone to estradiol $(\mathrm{p}=0.007)$. There were trends for associations between better verbal episodic memory and lower total testosterone $(\mathrm{p}=0.08)$ and lower testosterone/estradiol ratio $(\mathrm{p}=0.06)$. Lower free testosterone levels were associated with greater two-year improvement on verbal episodic memory $(\mathrm{p}=0.04)$; higher testosterone/estradiol predicted greater semantic memory improvement $(\mathrm{p}=0.03)$. In postmenopausal midlife women, endogenous estradiol and testosterone levels and the testosterone/estradiol ratio are associated with semantic memory and verbal episodic memory abilities.
\end{abstract}

Key words: cognition, estradiol, estrone, menopause, memory, testosterone 


\section{Introduction}

The extent to which, and the circumstances under which, endogenous and exogenous ovarian steroids affect cognitive function after natural menopause remains controversial. Circulating levels of estradiol decline as women enter the menopausal transition and drop to a low, steady level during the early postmenopause (Burger et al., 2007). In contrast, although testosterone declines during a woman's reproductive years, levels are generally unaffected by the natural menopausal transition (Burger et al., 2000; Davison et al., 2005). Memory complaints are highly prevalent during this midlife period (Woods et al., 2000). Some observational studies support a positive association between use of estrogen-containing hormone therapy and cognitive function in healthy women, but the results of randomized controlled trials have failed to show clear evidence of benefit (Henderson and Sherwin, 2007; Lethaby et al., 2008), and there is even some suggestion of mild detriment (Espeland et al., 2004; Maki et al., 2007). While this topic remains one of ongoing debate, the basic question of whether endogenous serum levels of these hormones influence cognitive performance remains uncertain.

In older postmenopausal women, relatively higher serum estradiol levels have been associated with better global cognitive function (Yaffe et al., 2000; Lebrun et al., 2005), episodic memory (Drake et al., 2000; Wolf and Kirschbaum, 2002; Hogervorst et al., 2004) and executive function (Wolf and Kirschbaum, 2002); however, findings are not consistent (Barrett-Connor and Goodman-Gruen, 1999; Carlson and Sherwin, 2000; Hogervorst et al., 2004). Relatively low estradiol levels in the late postmenopause have been linked to Alzheimer's disease risk in women (Manly et al., 2000; Hoskin et al., 2004), but again there are inconsistencies (Geerlings et al., 2003).

Few studies have investigated potential links between serum estrogens and cognitive function in recently postmenopausal women, even though the midlife period represents the time when women are most likely to use hormone therapy. Moreover, for this age group, studies have failed to find significant associations between cognitive function and total estradiol levels (Henderson et al., 2003; Herlitz et al., 2007; Luetters et al., 2007); associations with free estradiol are not reported. Most estradiol circulates tightly bound 
to sex hormone binding globulin (SHBG) or loosely bound to albumen. Free estradiol, which readily crosses the blood-brain barrier, is considered to be the biologically active form. Further, while the association between estrogen levels and cognitive function remains of strong interest, other gonadal hormones such as testosterone may also be important (Drake et al., 2000; Wolf and Kirschbaum, 2002; Thilers et al., 2006; Hogervorst et al., 2010). Receptors for estrogen and androgen are found in distinct neuronal populations (Simerly et al., 1990), and laboratory studies indicate that both estrogens and androgens are neuroprotective and can influence brain function (Spencer et al., 2008; Pike et al., 2009). Moreover, estrogen and androgen receptors may interact directly, and expression of each may be influenced by estrogens and by androgens (Adesanya-Famuyiwa et al., 1999; Panet-Raymond et al., 2000; Sharma and Thakur, 2006).

Our study sought to determine whether endogenous levels of estrone, estradiol, or testosterone are associated with specific domains of cognitive function in postmenopausal midlife women. We were particularly interested in associations with verbal episodic memory, a cognitive domain where poor performance is especially linked to increased dementia risk (Chen et al., 2000; Small et al., 2000). Total and free estradiol and testosterone levels, the ratio of these two hormones, and estrone were tested for association with cognition and with cognitive change over a two-year period.

\section{Methods}

\subsection{Study population}

Sampling procedures used to establish the Melbourne Women's Midlife Health Project cohort have been described (Dennerstein et al., 1993; Ryan et al., 2009). In brief, households were selected via random digital telephone dialing, and eligible women (Australian-born, aged 45-55 years and currently residing in Melbourne) provided written informed consent if they agreed to participate. The study protocol was approved by the University of Melbourne Human Research Ethics Committee, and procedures were followed in accordance with ethical standards of the National Health and Medical Research Council. Women who had 
menstruated in the three prior months, were not currently using oral contraceptives or hormone therapy, and had a uterus and at least one ovary were invited to participate in the longitudinal cohort. Compared with the rest of the survey population, the 438 women who agreed (response rate 56\%) reported better self-rated health and were more likely to have a tertiary education, to exercise at least once a week, to have ever had a Papanicolaou smear, and to have undergone dilatation and curettage (Burger et al., 1995).

In years 11 and 13, cohort members were administered a comprehensive neuropsychological battery to assess potential influences of reproductive status, hormone therapy, and other exposures on midlife cognitive skills. Although 317 women (85\%) remained in the longitudinal cohort at the time of initial testing in year 11 (i.e., baseline neuropsychological testing), present analyses were restricted to a sub-sample of 148 women. We excluded women if they did not participate in baseline cognitive testing $(n=60)$, were not yet postmenopausal $(n=22)$, reported surgical menopause $(n=40)$, were current hormone therapy users $(n=33)$, or were missing data for hormone levels $(n=7)$ or other covariates $(n=7)$.

\subsection{Cognitive testing}

The neuropsychological test battery was administered by two graduate psychologists. Tests included an adaption of the California Verbal Learning Test II (CVLT) (Delis et al., 2000) using semantically related words and a 10-item list learning task using unrelated words (Collie et al., 1999); the unrelated word list had been administered three years earlier as a solitary task (Henderson et al., 2003). Both tests assessed immediate (sum of 3 learning trials) and delayed recall. Immediate and delayed recognition trials (Faces I and II) from the Wechsler Memory Scale III were also administered (Wechsler, 1997). Other tasks were Letter-Number Sequencing and Block Design tests of the Wechsler Adult Intelligence Scale III (Wechsler, 1991), category fluency (animal naming) (Troyer, 2000), a 30-item version of the Boston Naming Test (Mack et al., 1992), the oral Symbol-Digit Modalities test (Smith, 1991), the Trail Making Test Part B (Reitan, 1958), and Judgment of Line Orientation (Benton et al., 1978). Details regarding test administration and 
normative values in this population have been reported (Clark et al., 2004b; 2004a; Elkadi et al., 2006b; 2006a).

\subsection{Hormone levels}

Postmenopausal women provided fasting morning blood samples at the time of baseline neuropsychological testing. Blood samples collected in EDTA tubes were centrifuged and the serum stored immediately at $-20^{\circ} \mathrm{C}$. Samples were transferred and stored for up to 1.5 years at $-80^{\circ} \mathrm{C}$. Estrone, estradiol, testosterone, and SHBG were measured by validated immunoassays optimized for measurements in women.

\subsubsection{Estrone, total estradiol, and total testosterone}

Serum was extracted with hexane-ethyl acetate (3:2) and extract purified by Celite column partition chromatography before radioimmunoassay, using ethylene glycol as the stationary phase (Goebelsmann et al., 1974; Goebelsmann et al., 1979). Elution of estrone from the column was carried out with $15 \%$ ethyl acetate in isooctane. Assay sensitivity is $5 \mathrm{pg} / \mathrm{ml}$; intraassay $\mathrm{CV}$ is $7.9 \%$ at $26 \mathrm{pg} / \mathrm{ml}$; and interassay $\mathrm{CV}$ is $12 \%$ at 26 $\mathrm{pg} / \mathrm{ml}$. Estradiol was eluted with $40 \%$ ethyl acetate in isooctane. Assay sensitivity is $4 \mathrm{pg} / \mathrm{ml}$; intraassay CV is $8.9 \%$ at $14 \mathrm{pg} / \mathrm{ml}$; and interassay $\mathrm{CV}$ is $14 \%$ at $14 \mathrm{pg} / \mathrm{ml}$. Testosterone was eluted with $40 \%$ toluene in isooctane. Assay sensitivity is $1.5 \mathrm{ng} / \mathrm{dl}$; the intraassay coefficient of variation $(\mathrm{CV})$ is $7.0 \%$ at $14.3 \mathrm{ng} / \mathrm{dl}$; and interassay $\mathrm{CV}$ is $10.4 \%$ at $6.1 \mathrm{ng} / \mathrm{dl}$.

\subsection{2. $S H B G$}

Solid-phase, two-site chemiluminescent immunoassay on the Immulite analyzer (Siemens Medical Solutions Diagnostics, Los Angeles, CA) was used to measure SHBG. Sensitivity is $1 \mathrm{nmol} / \mathrm{l}$; intraassay and interassay CVs are $4.1 \%$ at $7.7 \mathrm{nmol} / \mathrm{l}$ and $5.8 \%$ at $13 \mathrm{nmol} / \mathrm{l}$, respectively. 


\subsubsection{Free estradiol and free testosterone}

Free (nonprotein bound) estradiol and testosterone were calculated using a validated algorithm (Rinaldi et al., 2002) based on derived equations (Södergård et al., 1982; Vermeulen et al., 1999). The algorithm uses measured concentrations of total estradiol or testosterone and SHBG, an assumed average concentration of albumin $(3.5 \mathrm{~g} / \mathrm{dL})$, and appropriate affinity constants of SHBG and albumin for estradiol or testosterone. The molar ratio of free testosterone to free estradiol was also calculated (free testosterone, nmol/l divided by free estradiol, nmol/l) $\left(\mathrm{T} / \mathrm{E}_{2}\right)$, which is almost identical to the molar ratio of total testosterone to total estradiol.

\subsection{Other measures}

Questionnaires administered at each follow-up interview gathered information on socio-demographic and lifestyle characteristics, aging-related problems and overall health. Age at natural menopause defined by 12 months of amenorrhea was calculated based on last reported menstruation, in most instances validated by menstrual diaries (Taffe and Dennerstein, 2000). Body mass index $\left(\mathrm{kg} / \mathrm{m}^{2}\right)$ was calculated from measured height and weight. Information was recorded on self-reported education level, marital situation, smoking status, alcohol consumption over the last week and paid employment. Current level of physical activity was recorded as the frequency of engaging in exercise for fitness or recreational purposes (Netz et al., 2008). Women indicated whether they suffered from hot flushes or night sweats over the last two weeks. Chronic health conditions were identified based on physical measurements, self-report, and medication use. These included hypercholesterolemia, diabetes, hypertension, chronic asthma, heart disease, stomach or bowel ulcers, arthritis, rheumatism, cancer or migraine. A dichotomous variable was created to indicate whether a woman suffered from at least three of these health problems. Depressive symptoms were assessed using the 10-item Center for Epidemiology Studies Depression Scale (Radloff, 1977), and women scoring at least 10 from a maximum score of 30 were classified as having depressive symptoms (Andresen et al., 1994). Apolipoprotein E genotyping based on restriction enzyme isotyping was carried out from blood samples as 
previously described (Hixson and Vernier, 1990) at the University of Southern California (Los Angeles, CA), and participants carrying at least one copy of the $\varepsilon 4$ allele of the apolipoprotein E gene (APOE) were identified.

\subsection{Statistical Analysis}

To reduce the number of cognitive outcomes examined and thus the risk of a type 1 error, principal component analysis was performed to identify groupings of correlated cognitive tests. The principal axis method was used to extract the components (eigenvalues $>1$ ), followed by a varimax rotation. In interpreting the rotated factor pattern, a test was said to load on a given component if the factor loading was .50 or greater for that component. An individual's factor-based score on each of the components so identified was calculated by converting the scores for each specific cognitive test to a normalized z-score using the group means and standard deviations, and then averaging individual z-scores within each component. As the Trail Making Test is a timed task, the scoring for this variable was reversed before normalization and averaging, so higher scores indicated better performance on all tests (recoded score $=241$ minus original score, where 241 seconds $=$ maximum time recorded +1 ). The change in cognitive function over the two year period was calculated by subtracting each baseline test score from the corresponding score two years later. Z-scores were then derived for changes in individual test scores, and an average change score for each cognitive factor was calculated. Low change scores indicated a decline in cognitive function.

Initial analyses compared the characteristics of women at the time of baseline testing according to cognitive factor scores, using correlations and t-tests. Linear regression analysis was then used to model the association between individual hormone measures and cognitive function at baseline. The distribution of hormone levels could be approximately normalized using a log transformation. Analyses were adjusted $a$ priori for factors previously reported to confound the association between hormone measures and cognition: age, education level, depressive symptoms and age at menopause. Analyses also considered the inclusion of additional covariates. For 108 women who underwent cognitive testing at both time points, similar adjusted 
analyses controlling also for baseline cognitive scores, examined the association between baseline hormone measures and the two-year change in cognitive function. Two-way interaction terms were considered for hormone levels and age and education level. SAS version 9.1 (SAS Institute, Cary, NC) was used for statistical analyses with a two-tailed significance level of alpha less than 0.05 .

\section{Results}

\subsection{Cross-sectional analyses}

Characteristics of participants are summarized in Table 1, and hormone values are given in Table 2. Levels of estrone, total and free estradiol, and total and free testosterone were significantly, positively correlated (Table 3). The women had a mean age of 60 years; only six women were aged 65 or older. The average age at natural menopause was 53. Participants were in relatively good health. One third reported vasomotor symptoms, and the prevalence of depressive symptoms was low. Serum hormone levels were indicative of a postmenopausal population. Compared to women in the year 11 cohort not included in this baseline analysis, participants were older $(\mathrm{p}=0.02)$, with lower levels of total estradiol $(\mathrm{p}<0.001)$ and free estradiol $(\mathrm{p}<0.001)$, and higher values for total $(\mathrm{p}=0.05)$ and free testosterone $(\mathrm{p}<0.001)$ and for $\mathrm{T} / \mathrm{E}_{2}$ $(\mathrm{p}<0.001)$. These observations reflect exclusion of pre- and peri-menopausal women. Included women performed better on the verbal episodic memory factor ( $\mathrm{p}=0.04$; see below) compared to excluded women but did not differ on other cognitive test results or general participant characteristics.

Principal component analysis of neuropsychological test scores resulted in a four-component solution, accounting for $63 \%$ of the variance. The identified cognitive factors were labeled as verbal episodic memory, visual episodic memory, semantic memory, and executive function-visuospatial skills (Table 4). Chronbach's alphas for component tests were 0.77 (factor 1), 0.73 (factor 2), 0.60 (factor 3), and 0.75 (factor

4). Factors were significantly correlated with one another ( $r$ values 0.27 to $0.34, p<0.001$ ) with the exception of the correlation between visual memory and executive-visuospatial factors ( $r=0.13, p=0.12$ ). 
In linear regression models adjusted for age, age at menopause, education level, and depressive symptoms, a significant positive association was found between both total and free log estradiol levels and the semantic memory factor (Table 5). A highly significant negative association was also found between $\log \mathrm{T} / \mathrm{E}_{2}$ and this factor, indicating that a higher level of free testosterone relative to free estradiol was associated with worse performance on semantic memory tasks. There were also trends towards higher values of $\log \mathrm{T} / \mathrm{E}_{2}$ $(\mathrm{p}=0.06)$ and total testosterone $(\mathrm{p}=0.08)$ being associated with worse verbal episodic memory. No significant associations were found between any hormone value and either visual episodic memory or executivevisuospatial function. Controlling for covariates from Table 1 in these models did not modify the results. Removing mood from the regression models had essentially no effect on significant or near-significant associations reported in Table 5, and no new significant associations were revealed (data not shown). SHBG showed no independent association with any cognitive factor (p's $>0.4$ ). Effects were not modified by age or education.

To assess further these identified associations, post-hoc analyses explored the relationship between hormone levels and the individual cognitive tests within these factors. With respect to the semantic memory factor, total and free estradiol (positively), as well as $\mathrm{T} / \mathrm{E}_{2}$ (negatively), were significantly associated with performance on the Boston Naming Test (Supplementary Table 1A). T/E $E_{2}$ was also significantly negatively associated with category fluency scores. The trends for associations between total and free testosterone levels and $\mathrm{T} / \mathrm{E}_{2}$ values with verbal episodic memory resulted from negative associations with CVLT immediate and delayed recall scores (Supplementary Table 1B).

\subsection{Longitudinal analyses}

Change in cognitive function was assessed after a two year follow-up period. Mean scores on all tests except the Boston Naming Test and Letter-Number Sequencing improved between the two test sessions, and the mean length of time to complete the Trail Making Test increased slightly. Both higher total and free testosterone levels were associated with smaller mean improvement on the verbal episodic memory factor 
(Table 6), in particular the immediate and delayed recall tasks of the unrelated word list (Supplementary Table 2). There was also a significant positive association between $\mathrm{T} / \mathrm{E}_{2}$ and the change in semantic memory performance over time (Table 6); post hoc analysis showed only weak associations with the two neuropsychological tests comprising this factor (both $\mathrm{p}$ values $=0.16$; data not shown). Removing mood from the regression models had essentially no effect on significant or near-significant associations in Tables 6, and no new significant associations were revealed (data not shown). SHBG was not independently associated with change in cognitive factors ( $\mathrm{p}$ 's $>0.1$ ). Effects were not modified by age or education.

\section{Discussion}

\subsection{Relations between hormone measures and cognitive outcomes}

In this well characterized, relatively young postmenopausal cohort, we examined associations between sex hormones - serum levels of estrone, total and free estradiol, and total and free testosterone, as well as $\mathrm{T} / \mathrm{E}_{2}$ - and four domains of cognitive function. In both cross-sectional and longitudinal analyses, principal findings concerned two of these domains: semantic memory and verbal episodic memory. There were no significant associations with hormone measures and visual episodic memory or executivevisuospatial factors. Estrone and SHBG were unrelated to cognitive measures. Associations with cognition were similar for free and total hormone levels, as these values were highly correlated.

Better semantic memory was associated with higher estradiol levels (total and free) and a lower ratio of testosterone to estradiol. There were also trends for higher total testosterone and higher $\mathrm{T} / \mathrm{E}_{2}$ to be associated with lower verbal episodic memory. Findings in two-year longitudinal analyses showed both similarities and differences when compared to cross-sectional results. Higher total and free testosterone levels were associated with less improvement on verbal episodic memory, a finding that supports trends seen with baseline cognitive scores. There were no significant associations between estrogen levels and cognitive 
change. Higher $\mathrm{T} / \mathrm{E}_{2}$ at baseline was associated with greater mean improvement in semantic memory over two years, which contrasts with cross-sectional findings of an inverse association between $\mathrm{T} / \mathrm{E}_{2}$ and semantic memory. This apparent discrepancy may be partially understood if relevant hormonal effects on brain function and thus cognitive performance are recognized as "activational" (and thus relatively short lived) rather than "organizational" (Arnold, 2009). Hormone levels at one point in time may have limited predictive value for performance two years later, except to the extent that current levels correlate with future levels. Practice and ceiling effects may also have played roles. Mean scores on almost all tests improved over the two years, and practice effects made it more difficult to discern cognitive decline. Ceiling effects of one of the semantic memory tasks (Boston Naming Test) limited the ability of strong performers at baseline to improve scores two years later.

In prior analyses from the Melbourne Women's Midlife Health Project cohort, we found no association between word-list learning for unrelated words and serum levels of total estradiol or the free estradiol index (Henderson et al., 2003). Two subsequent studies of midlife women (Herlitz et al., 2007; Luetters et al., 2007) similarly failed to find significant associations between total estradiol and verbal episodic memory or verbal fluency. Our present findings involving free estradiol strengthen these earlier findings. None of these studies considered testosterone levels.

Other investigators have examined associations between estrogen levels and cognitive function in older postmenopausal women, with conflicting results (Barrett-Connor and Goodman-Gruen, 1999; Drake et al., 2000; Wolf and Kirschbaum, 2002). Likewise for testosterone, reports in older women, or in a mixed population of younger and older postmenopausal women, describe positive (Barrett-Connor and GoodmanGruen, 1999; Carlson and Sherwin, 2000; Drake et al., 2000; Wolf and Kirschbaum, 2002; Hogervorst et al., 2010), negative (Hogervorst et al., 2004; Thilers et al., 2006) and null associations with cognitive function (Carlson and Sherwin, 2000; Drake et al., 2000; Hogervorst et al., 2004; Hogervorst et al., 2010). Variable results in part could be because levels of estradiol and testosterone were not considered together, because cognitive tasks differed, or because population characteristics differed. 
As estradiol levels (Burger et al., 2007) — but not testosterone levels (Burger et al., 2000; Davison et al., 2005) - decline dramatically across the natural menopausal transition, the ratio of testosterone to estradiol will increase. In our study, high levels of testosterone relative to estradiol were negatively associated with both semantic memory and verbal episodic memory. Moreover, total and free testosterone predicted poorer verbal episodic memory performance over a two year period. Although there were no significant or near-significant associations between hormone values and visual episodic memory, total testosterone and $\mathrm{T} / \mathrm{E}_{2}$ showed similar effect sizes for both verbal and visual episodic memory (Table 5), suggesting that apparent deleterious cross-sectional associations between episodic memory and testosterone or $\mathrm{T} / \mathrm{E}_{2}$ are not necessarily modality specific.

Many prior studies infer hormone effects on a particular cognitive domain on the basis of a single cognitive test. We found significant associations for estradiol levels (positive) and $\mathrm{T} / \mathrm{E}_{2}$ values (negative) with semantic memory, as well as negative trends for total testosterone and $\mathrm{T} / \mathrm{E}_{2}$ with verbal episodic memory; post hoc analyses enabled us to examine which specific tests within these cognitive domains were primarily responsible for these associations.

Semantic memory refers to memory for generic, over-learned information, including memory for word names. For the semantic memory factor, higher total estradiol, higher free estradiol and lower T/E $\mathrm{E}_{2}$ were each significantly associated with Boston Naming Test scores (Supplementary Table 1A). Lower T/E 2 values were significantly associated with category fluency. Trouble with word names is a common midlife complaint. Semantic memory involves widely distributed areas of cerebral cortex (Patterson et al., 2007). Estrogen receptor beta shows a wide distribution within layer II-VI cortical neurons (González et al., 2007), providing a hypothetical link between menopausal estrogen loss and semantic memory symptoms. The association between estradiol and naming suggests the need to examine more closely the effects of menopause on this particular cognitive ability.

For the verbal episodic memory factor, negative associations for total testosterone and $\mathrm{T} / \mathrm{E}_{2}$ were identified for immediate and delayed recall tasks of the CVLT, whereas there were no associations for unrelated word list recall tasks (Supplementary Table 1B). Although performances on these memory tasks 
are correlated $(\mathrm{r}=0.36$ and 0.29 for immediate and delayed recall tasks respectively, $\mathrm{p}$ values $<0.001)$, psychomotor properties of the two tests differ. The CVLT word list is longer than the unrelated word list (16 versus 10 words) and may, on this basis, provide a more precise estimate of episodic memory ability. Another key difference is that the CVLT is composed of groups of semantically related words. Recognizing the relation among CVLT words facilitates learning and recall, and implicates executive control processes in addition to memory skills. In fact, it is suggested that executive processing involving the prefrontal cortex is especially sensitive to estrogens (Joffe et al., 2006). However, we observed no significant associations between hormone measures and the executive-visuospatial factor in our cohort. It is interesting that negative associations between free and total testosterone and two-year changes in verbal episodic memory were stronger for recall of unrelated words (Supplementary Table 2). The unrelated word-list learning task had been previously administered to cohort members as a solitary measure (Henderson et al., 2003), and speculatively, relevant memory processes may have been more closely linked to the stronger practice (learning) effect than to word list acquisition and recall within the short temporal window encapsulated within a test session.

More generally, post hoc findings emphasize the inherent limitation of generalizing associations based on an individual neuropsychological test — performance of which inevitably draws to some extent upon multiple attentional, perceptual, processing, and executive skills — to an entire domain of cognitive function.

\subsection{Limitations and strengths}

The relatively modest sample size limits power to detect smaller associations between hormone measures and cognition, although sample sizes are larger than in many previous reports of postmenpausal women (Carlson and Sherwin, 2000; Drake et al., 2000; Wolf and Kirschbaum, 2002; Hogervorst et al., 2004). To reduce the likelihood of false-positive associations, primary analyses used principal component analysis to reduce the number of cognitive end points. Some associations with hormone measures, however, 
could still have occurred by chance. It would be injudicious to generalize cognitive findings in healthy postmenopausal midlife women — where serum levels of estrone and estradiol are relative low — to clinical populations (for example, women with frank dementia) or to women with hormone levels above the range included in our sample (for example, women of reproductive age or postmenopausal women receiving exogenous estrogens or testosterone). Data concerning some covariates were self-reported and thus may be subject to recall bias and misclassification. Bias may have been introduced through the exclusion of women who did not complete the cognitive testing or who used hormone therapy. In longitudinal analyses, we examined cognitive change over a two year period, and for most tests the average score had improved. This practice effect made it more difficult to detect associations between hormone levels and age-related cognitive decline, particularly as women were relatively young (mean age 60 years) and healthy. Because we did not measure hormone levels at the time of the second testing, we were unable to examine associations between hormone changes and cognitive change. Finally, there may have been unrecognized confounding factors that were not considered in these analyses.

This study has a number of strengths. This is a population-based study of randomly recruited women, and analyses adjusted for a number of factors that could potentially confound the association between hormone levels and cognitive function. We have considered not only levels of estrone, total estradiol and total testosterone but also free estradiol and testosterone levels and the ratio between levels of free testosterone and free estradiol. Further, our broadly based neuropsychological battery permitted examination of a number of cognitive domains impacted by cognitive aging, some of which are suggested as sentinel markers of late-life dementia.

\subsection{Concluding perspectives}

The relationships between endogenous sex steroids, brain function and behaviors are undoubtedly complex. Free steroid hormones rapidly cross the blood-brain barrier, and within the brain testosterone can be converted to estradiol by the enzyme aromatase (Boon et al., 2010). In addition, these hormones are 
neurosteroids (Mukai et al., 2006), subject to endogenous synthesis within the brain from cholesterol precursors. Serum levels may therefore reflect brain levels imprecisely.

Our results show that serum levels of estradiol or testosterone in isolation are less revealing than measures of both hormones. This finding is perhaps not surprising, given the potential for physiological interactions between estrogens and androgens on several levels (Adesanya-Famuyiwa et al., 1999; PanetRaymond et al., 2000; Sharma and Thakur, 2006; Boon et al., 2010). The balance between estradiol and testosterone may be therefore important for some aspects of cognitive function in postmenopausal midlife women. The finding that the relation between gonadal hormones and cognition differs across cognitive domains indicates that a single global measure of cognition may thus overlook important associations limited to a specific domain.

Results support the view that estradiol, within the range of endogenous levels examined in this study, is associated with better semantic memory — and perhaps naming ability more specifically — in postmenopausal midlife women.

Consistent with prior reports involving midlife and elderly women (Thilers et al., 2006) and surgically menopausal women (Möller et al., 2010), our results suggest also that relative androgen excess in midlife is negatively associated with performance on tasks of semantic and verbal episodic memory. Relatively high testosterone is linked to adverse cognitive outcomes in older women as well (Hogervorst et al., 2004). The association, while not necessarily causal, is of concern. Verbal episodic memory is sensitive to cognitive aging (Weintraub et al., 2009), and impaired performance in this domain is linked to development of Alzheimer's disease later in life (Chen et al., 2000; Small et al., 2000). Observed associations for endogenous testosterone levels do not necessarily predict effects of exogenous testosterone therapy administered at pharmacological doses, but findings suggest the need for further study when off-label testosterone therapy is considered for midlife women. Findings also suggest the need to consider the role of testosterone in designing and interpreting results of trials of estrogen therapy or estrogen-progestin therapy in midlife women. 
More generally, our results highlight the importance of considering the relation between estradiol and testosterone rather than each hormone in isolation, of assessing specific cognitive skills rather than global cognition, and of using more than a single neuropsychological test to define performance within a cognitive domain. This approach may help resolve inconsistencies in the literature regarding associations between gonadal hormones, cognition, and cognitive aging after a woman's reproductive years. 


\section{Disclosure statement}

JR, FZS, WJM, MSC, DK and VWH report no actual or potential conflicts of interest. LD has received lecture and consulting fees from Bayer Schering, Wyeth and Boehringer Ingelheim, and she has served on advisory boards for Wyeth, Boehringer Ingelheim and Bayer Schering. CS has received honoraria from Pfizer and Lundbeck.

\section{Acknowledgments}

Research in the Melbourne Women's Midlife Health Study was supported by grants from the Alzheimer's Association IIRG-01-2684 (VWH), Victorian Health Promotion Foundation (LD), the National Health and Medical Research Council of Australia (LD, CS), and the University of Melbourne. Effort of CS was supported by the Alzheimer's Association of Australia, Scobie and Claire McKinnon Foundation, and the Collier Trust Fund. We are indebted to the women in the Melbourne Women's Midlife Health Project, who generously donated their time to participate in this research study. 


\section{References}

Adesanya-Famuyiwa, O.O., Zhou, J., Wu, G. and Bondy, C., 1999. Localization and sex steroid regulation of androgen receptor gene expression in rhesus monkey uterus. Obstet. Gynecol. 93, 265-270.

Andresen, E.M., Malmgren, J.A., Carter, W.B. and Patrick, D.L., 1994. Screening for depression in well older adults: evaluation of a short form of the CES-D. Am. J. Prev. Med. 10, 77-84.

Arnold, A.P., 2009. The organizational-activational hypothesis as the foundation for a unified theory of sexual differentiation of all mammalian tissues. Horm. Behav. 55, 570-578.

Barrett-Connor, E. and Goodman-Gruen, D., 1999. Cognitive function and endogenous sex hormones in older women. J. Am. Geriatr. Soc. 47, 1289-1293.

Benton, A.L., Varney, N.R. and Hamsher, K., 1978. Visuospatial judgment: a clinical test. Arch. Neurol. 35, 364-367.

Boon, W.C., Chow, J.D. and Simpson, E.R., 2010. The multiple roles of estrogens and the enzyme aromatase. Prog. Brain Res. 181, 209-232.

Burger, H.G., Dudley, E.C., Hopper, J.L., Shelley, J.M., Green, A., Smith, A., Dennerstein, L. and Morse, C.M., 1995. The endocrinology of the menopausal transition: a cross-sectional study of a populationbased sample. J. Clin. Endocrinol. Metab. 80, 3537-3545.

Burger, H.G., Dudley, E.C., Cui, J., Dennerstein, L. and Hopper, J.L., 2000. A prospective longitudinal study of serum testosterone, dehydroepiandrosterone sulfate, and sex hormone-binding globulin levels through the menopause transition. J. Clin. Endocrinol. Metab. 85, 2832-2838.

Burger, H.G., Hale, G.E., Robertson, D.M. and Dennerstein, L., 2007. A review of hormonal changes during the menopausal transition: focus on findings from the Melbourne Women's Midlife Health Project. Hum. Reprod. Update 13, 559-565.

Carlson, L.E. and Sherwin, B.B., 2000. Higher levels of plasma estradiol and testosterone in healthy elderly men compared with age-matched women may protect aspects of explicit memory. Menopause 7, 168177.

Chen, P., Ratcliff, G., Belle, S.H., Cauley, J.A., DeKosky, S.T. and Ganguli, M., 2000. Cognitive tests that best discriminate between presymptomatic $\mathrm{AD}$ and those who remain nondemented. Neurology 55, 1847-1853.

Clark, M.S., Dennerstein, L., Elkadi, S., Guthrie, J.R., Bowden, S.C. and Henderson, V.W., 2004a. Normative verbal and non-verbal memory test scores for Australian women aged 56-67. Aust. N. Z. J. Psychiatry $38,532-540$.

Clark, M.S., Dennerstein, L., Elkadi, S., Guthrie, J.R., Bowden, S.C. and Henderson, V.W., 2004b. Normative data for tasks of executive function and working memory for Australian-born women aged 56-67. Aust. Psychol. 39, 244-250.

Collie, A., Shafiq-Antonacci, R., Maruff, P., Tyler, P. and Currie, J., 1999. Norms and the effects of demographic variables on a neuropsychological battery for use in healthy ageing Australian populations. Aust. N. Z. J. Psychiatry 33, 568-575.

Davison, S., Bell, R., Donath, S., Montalto, J. and Davis, S.R., 2005. Androgen levels in adult females: changes with age, menopause and oophorectomy. J. Clin. Endocrinol. Metab. 90, 3847-3853.

Delis, D.C., Kramer, J., Kaplan, E. and Ober, B.A., 2000. CVLT-II. California Verbal Learning Test, second edition, adult version. Harcourt-Brace, San Antonio, TX.

Dennerstein, L., Smith, A.M.A., Morse, C.A., Green, A., Burger, H. and Hopper, J.L., 1993. Menopausal symptoms in Australian women. Med. J. Aust. 159, 232-236.

Drake, E.B., Henderson, V.W., Stanczyk, F.Z., McCleary, C.A., Brown, W.S., Smith, C.A., Rizzo, A.A., Murdock, G.A. and Buckwalter, J.G., 2000. Associations between circulating sex steroid hormones and cognition in normal elderly women. Neurology 54, 599-603.

Elkadi, S., Clark, M.S., Dennerstein, L., Guthrie, J.R., Bowden, S.C. and Henderson, V.W., 2006a. Normative visuospatial performance in Australian midlife women. Aust. Psychol. 41, 43-47. 
Elkadi, S., Clark, M.S., Dennerstein, L., Guthrie, J.R., Bowden, S.C. and Henderson, V.W., 2006b. Normative data for Australian midlife women on category fluency and a short form of the Boston Naming Test. Aust. Psychol. 41, 37-42.

Espeland, M.A., Rapp, S.R., Shumaker, S.A., Brunner, R., Manson, J.E., Sherwin, B.B., Hsia, J., Margolis, K.L., Hogan, P.E., Wallace, R., Dailey, M., Freeman, R., Hays, J. and WHIMS Investigators, 2004. Conjugated equine estrogens and global cognitive function in postmenopausal women: Women's Health Initiative Memory Study. JAMA 291, 2959-2968.

Geerlings, M.I., Launer, L.J., de Jong, F.H., Ruitenberg, A., Stijnen, T., van Swieten, J.C., Hofman, A., Witteman, J.C.P., H A and Breteler, M.M., 2003. Endogenous estradiol and risk of dementia in women and men: the Rotterdam Study. Ann. Neurol. 53, 607-615.

Goebelsmann, U., Arce, J.J., Thorneycroft, I.H. and Mishell, D.R., Jr, 1974. Serum testosterone concentrations in women throughout the menstrual cycle and following HCG administration. Am. J. Obstet. Gynecol. 119, 445-452.

Goebelsmann, U., Bernstein, G.S., Gale, J.A., Kletzky, O.A., Nakamura, R.M., Coulson, A.H. and Korelitz, J.J., 1979. Serum gonadotropin, testosterone, estradiol and estrone levels prior to and following bilateral vasectomy, in: I.H. Lepow and R. Crozier (Eds.), Vasectomy: Immunologic and Pathophysiologic Effects in Animals and Man. Academic Press, New York, pp. 165-175.

González, M., Cabrera-Socorro, A., Pérez-García, C.G., Fraser, J.D., López, F.J., Alonso, R. and Meyer, G., 2007. Distribution patterns of estrogen receptor alpha and beta in the human cortex and hippocampus during development and adulthood. J. Comp. Neurol. 503, 790-802.

Henderson, V.W., Dudley, E.C., Guthrie, J.R., Burger, H.G. and Dennerstein, L., 2003. Estrogen exposures and memory at midlife: a population-based study of women. Neurology 60, 1369-1371.

Henderson, V.W. and Sherwin, B.B., 2007. Surgical versus natural menopause: cognitive issues. Menopause $14,572-579$.

Herlitz, A., Thilers, P. and Habib, R., 2007. Endogenous estrogen is not associated with cognitive performance before, during, or after menopause. Menopause 14, 425-431.

Hixson, J.E. and Vernier, D.T., 1990. Restriction isotyping of human apolipoprotein E by gene amplification and cleavage with HhaI. J. Lipid Res. 31, 545-548.

Hogervorst, E., De Jager, C., Budge, M. and Smith, A.D., 2004. Serum levels of estradiol and testosterone and performance in different cognitive domains in healthy elderly men and women. Psychoneuroendocrinology 29, 405-421.

Hogervorst, E., Matthews, F.E. and Brayne, C., 2010. Are optimal levels of testosterone associated with better cognitive function in healthy older women and men? Biochim. Biophys. Acta [Epub ahead of print].

Hoskin, E.K., Tang, M.X., Manly, J.J. and Mayeux, R., 2004. Elevated sex-hormone binding globulin in elderly women with Alzheimer's disease. Neurobiol. Aging 25, 141-147.

Joffe, H., Hall, J.E., Gruber, S., Sarmiento, I.A., Cohen, L.S., Yurgelun-Todd, D. and Martin, K.A., 2006. Estrogen therapy selectively enhances prefrontal cognitive processes: a randomized, double-blind, placebo-controlled study with functional magnetic resonance imaging in perimenopausal and recently postmenopausal women. Menopause 13, 411-422.

Lebrun, C.E., van der Schouw, Y.T., H, d.J.F., Pols, H.A., Grobbee, D.E. and Lamberts, S.W., 2005. Endogenous oestrogens are related to cognition in healthy elderly women. Clin. Endocrinol. (Oxf). $63,50-55$.

Lethaby, A., Hogervorst, E., Richards, M., Yesufu, A. and Yaffe, K., 2008. Hormone replacement therapy for cognitive function in postmenopausal women. Cochrane Database Syst. Rev. Issue 1, Art. No.: CD003122. DOI: 003110.001002/14651858.CD14003122.pub14651852.

Luetters, C., Huang, M.H., Seeman, T., Buckwalter, G., Meyer, P.M., Avis, N.E., Sternfeld, B., Johnston, J.M. and Greendale, G.A., 2007. Menopause transition stage and endogenous estradiol and folliclestimulating hormone levels are not related to cognitive performance: cross-sectional results from the study of women's health across the nation (SWAN). J. Womens Health 16, 331-344.

Mack, W.J., Freed, D.M., Williams, B.W. and Henderson, V.W., 1992. Boston Naming Test: shortened versions for use in Alzheimer's disease. J. Gerontol. 47, P154-P158. 
Maki, P.M., Gast, M.J., Vieweg, A., Burriss, S.W. and Yaffe, K., 2007. Hormone therapy in menopausal women with cognitive complaints: a randomized, double-blind trial. Neurology 69, 1322-1330.

Manly, J.J., Merchant, C.A., Jacobs, D.M., Small, S.A., Bell, K., Ferin, M. and Mayeux, R., 2000. Endogenous estrogen levels and Alzheimer's disease among postmenopausal women. Neurology 54, 833-837.

Möller, M.C., Bartfai, A.B. and Rådestad, A.F., 2010. Effects of testosterone and estrogen replacement on memory function. Menopause 17, Epub ahead of print.

Mukai, H., Tsurugizawa, T., Ogiue-Ikeda, M., Murakami, G., Hojo, Y., Ishii, H., Kimoto, T. and Kawato, S., 2006. Local neurosteroid production in the hippocampus: influence on synaptic plasticity of memory. Neuroendocrinology 84, 255-263.

Netz, Y., Zach, S., Taffe, J.R., Guthrie, J. and Dennerstein, L., 2008. Habitual physical activity is a meaningful predictor of well-being in mid-life women: a longitudinal analysis. Climacteric 11, 337344.

Panet-Raymond, V., Gottlieb, B., Beitel, L.K., Pinsky, L. and Trifiro, M.A., 2000. Interactions between androgen and estrogen receptors and the effects on their transactivational properties. Mol. Cell. Endocrinol. 167, 139-150.

Patterson, K., Nestor, P. and Rogers, T.T., 2007. Where do you know what you know? The representation of semantic knowledge in the human brain. Nat. Rev. Neurosci. 8, 976-987.

Pike, C.J., Carroll, J.C., Rosario, E.R. and Barron, A.M., 2009. Protective actions of sex steroid hormones in Alzheimer's disease. Front. Neuroendocrinol. 30, 239-258.

Radloff, L.S., 1977. The CES-D scale: a self-report depression scale for research in the general population. Appl. Psychol. Meas. 1, 385-401.

Reitan, R.M., 1958. Validity of the trail Making Test as an indicator of organic brain damage. Percept. Mot. Skills 8, 271-276.

Rinaldi, S., Geay, A., Déchaud, H., Biessy, C., Zeleniuch-Jacquotte, A., Akhmedkhanov, A., Shore, R.E., Riboli, E., Toniolo, P. and Kaaks, R., 2002. Validity of free testosterone and free estradiol determinations in serum samples from postmenopausal women by theoretical calculations. Cancer Epidemiol. Biomarkers Prev. 11, 1065-1071.

Ryan, J., Burger, H.G., Szoeke, C., Lehert, P., Ancelin, M.L., Henderson, V.W. and Dennerstein, L., 2009. A prospective study of the association between endogenous hormones and depressive symptoms in postmenopausal women. Menopause 16, 509-517.

Sharma, P.K. and Thakur, M.K., 2006. Expression of estrogen receptor (ER) $\alpha$ and $\beta$ in mouse cerebral cortex: effect of age, sex and gonadal steroids. Neurobiol. Aging 27, 880-887.

Simerly, R.B., Chang, C., Muramatsu, M. and Swanson, L.W., 1990. Distribution of androgen and estrogen receptor mRNA-containing cells in the rat brain: an in situ hybridization study. J. Comp. Neurol. 294, 76-95.

Small, B.J., Fratiglioni, L., Viitanen, M., Winblad, B. and Bäckman, L., 2000. The course of cognitive impairment in preclinical Alzheimer disease. Arch. Neurol. 57, 839-844.

Smith, A., 1991. Symbol Digit Modalities Test Manual. Western Psychological Services, Los Angeles.

Södergård, R., Bäckström, T., Shanbhag, V. and Carstensen, H., 1982. Calculation of free and bound fractions of testosterone and estradiol-17 beta to human plasma proteins at body temperature. J. Steroid Biochem. 16, 801-810.

Spencer, J.L., Waters, E.M., Romeo, R.D., Wood, G.E., Milner, T.A. and McEwen, B.S., 2008. Uncovering the mechanisms of estrogen effects on hippocampal function. Front. Neuroendocrinol. 29, 219-237.

Taffe, J. and Dennerstein, L., 2000. Retrospective self-report compared with menstrual diary data prospectively kept during the menopausal transition. Climacteric 3, 183-191.

Thilers, P.P., Macdonald, S.W. and Herlitz, A., 2006. The association between endogenous free testosterone and cognitive performance: a population-based study in 35 to 90 year-old men and women. Psychoneuroendocrinology 31, 565-576.

Troyer, A.K., 2000. Normative data for clustering and switching on verbal fluency tasks. J. Clin. Exp. Neuropsychol. 22, 370-378. 
Vermeulen, A., Verdonck, L. and Kaufman, J.M., 1999. A critical evaluation of simple methods for the estimation of free testosterone in serum. J. Clin. Endocrinol. Metab. 84, 3666-3672.

Wechsler, D., 1991. Wechsler Adult Intelligence Scale - III. Psychological Corporation, New York.

Wechsler, D., 1997. WMS-III. Wechsler Memory Scale - third edition. Psychological Corporation, San Antonio.

Weintraub, S., Salmon, D., Mercaldo, N., Ferris, S., Graff-Radford, N.R., Chui, H., Cummings, J., DeCarli, C., Foster, N.L., Galasko, D., Peskind, E., Dietrich, W., Beekly, D.L., Kukull, W.A. and Morris, J.C., 2009. The Alzheimer's Disease Centers' Uniform Data Set (UDS): the neuropsychologic test battery. Alzheimer Dis. Assoc. Disord. 23, 91-101.

Wolf, O.T. and Kirschbaum, C., 2002. Endogenous estradiol and testosterone levels are associated with cognitive performance in older women and men. Horm. Behav. 41, 259-266.

Woods, N.F., Mitchell, E.S. and Adams, C., 2000. Memory functioning among midlife women: observations from the Seattle Midlife Women's Health Study. Menopause 7, 257-265.

Yaffe, K., Lui, L.Y., Grady, D., Cauley, J., Kramer, J. and Cummings, S.R., 2000. Cognitive decline in women in relation to non-protein-bound oestradiol concentrations. Lancet 356, 708-712. 
Table 1. Participant characteristics $(n=148)$

Clinical or demographic feature ${ }^{a}$

Age, years
Age at menopause, years
Years since menopause
Body mass index, $\mathrm{kg} / \mathrm{m}^{2}$

At least 12 years of education

Married or living with a partner

Current smoker

Weekly alcohol consumption

None

$1-7$ drinks per week

8 or more drinks per week

Current paid employment

Full Time

Part time / casual / occasional

Not working

Current physical activity

Less than a few times each month

$1-3$ times per week

At least 4 times each week

Hot flushes or night sweats, past two weeks

Past user of hormone therapy

Chronic health problems

Depressive symptoms

At least one copy of the $A P O E \& 4$ allele
Value

$\begin{array}{ll}\text { Mean (SD) } & \text { Range } \\ 60(2.4) & 56-67 \\ 53(2.4) & 47-62 \\ 6.8(2.3) & 1.1-11.2 \\ 27(5.3) & 17-47\end{array}$

\section{Number}

\section{Percent}

54

37

$113 \quad 76$

9

6

26

18

$80 \quad 54$

42

28

75

51

54

37

19

13

21

14

59

40

$68 \quad 46$

$52 \quad 35$

$52 \quad 35$

$24 \quad 16$

$28 \quad 19$

$49 \quad 34$

${ }^{\mathrm{a}}$ See text for details. 
Table 2. Serum hormones values and sex hormone binding globulin levels $(n=148)$

\begin{tabular}{llll}
\hline Measure & Median & Interquartile range & Reference intervals \\
\hline Sex hormone binding globulin, nmol/1 & 42.7 & $31.8-57.0$ & $17-95$ \\
Estrone, pg/ml & 16.1 & $11.0-22.6$ & $10-60$ \\
Total estradiol, pg/ml & 13.4 & $10.7-16.4$ & $<30$ \\
Free estradiol, pg/ml & 0.33 & $0.26-0.45$ & $0.06-0.75$ \\
Total testosterone, ng/dl & 24.6 & $19.7-32.6$ & $5-50$ \\
Free testosterone, pg/ml & 5.2 & $3.8-6.3$ & $0.6-6.7$ \\
Free testosterone / free estradiol, molar ratio & 18.1 & $13.7-23.1$ & - \\
\hline
\end{tabular}


Table 3. Pearson correlations and probability values among serum hormone values and sex hormone binding globulin (n=148) ${ }^{\mathrm{a}}$

\begin{tabular}{|c|c|c|c|c|c|c|}
\hline & Estrone & $\begin{array}{l}\text { Total } \\
\text { estradiol }\end{array}$ & $\begin{array}{l}\text { Free } \\
\text { estradiol }\end{array}$ & $\begin{array}{l}\text { Total } \\
\text { testosterone }\end{array}$ & $\begin{array}{l}\text { Free } \\
\text { testosterone }\end{array}$ & $\begin{array}{l}\text { Free testosterone / free } \\
\text { estradiol, molar ratio }\end{array}$ \\
\hline \multirow[t]{2}{*}{ Sex hormone binding globulin } & 0.19 & -0.27 & -0.61 & 0.16 & -0.36 & 0.37 \\
\hline & 0.02 & 0.0009 & $<0.0001$ & 0.06 & $<0.0001$ & $<0.0001$ \\
\hline \multirow[t]{2}{*}{ Estrone } & & 0.65 & 0.60 & 0.35 & 0.41 & -0.23 \\
\hline & & $<0.0001$ & $<0.0001$ & $<0.0001$ & $<0.0001$ & 0.008 \\
\hline \multirow[t]{2}{*}{ Total estradiol } & & & 0.92 & 0.39 & 0.49 & -0.40 \\
\hline & & & $<0.0001$ & $<0.0001$ & $<0.0001$ & $<0.0001$ \\
\hline \multirow[t]{2}{*}{ Free estradiol } & & & & 0.26 & 0.55 & -0.46 \\
\hline & & & & 0.001 & $<0.0001$ & $<0.0001$ \\
\hline \multirow[t]{2}{*}{ Total testosterone } & & & & & 0.86 & 0.69 \\
\hline & & & & & $<0.0001$ & $<0.0001$ \\
\hline \multirow[t]{2}{*}{ Free testosterone } & & & & & & 0.48 \\
\hline & & & & & & $<0.0001$ \\
\hline
\end{tabular}

${ }^{\mathrm{a}}$ Correlations are based on $\log$ values. 
Table 4. Neuropsychological battery of tests, by cognitive factor $(n=148)$

\begin{tabular}{|c|c|c|c|}
\hline Cognitive factor & Test & Mean (SD) & Range \\
\hline \multirow[t]{4}{*}{ Verbal episodic memory } & CVLT, immediate recall & $26.8(5.8)$ & $13-39$ \\
\hline & CVLT, delayed recall & $9.0(3.3)$ & $0-16$ \\
\hline & Unrelated word list, immediate recall & $21.5(3.0)$ & $15-29$ \\
\hline & Unrelated word list, delayed recall & $5.8(2.2)$ & $0-10$ \\
\hline \multirow[t]{2}{*}{ Visual episodic memory } & Faces, immediate recognition & $35.1(4.3)$ & $25-44$ \\
\hline & Faces, delayed recognition & $36.6(4.6)$ & $20-46$ \\
\hline \multirow[t]{2}{*}{ Semantic memory } & Boston Naming Test & $27.4(2.1)$ & $21-30$ \\
\hline & Category fluency (animal naming) & $20.6(5.5)$ & $11-37$ \\
\hline Executive function & Trail Making Test, part B & $85.8(30.1)$ & $41-240$ \\
\hline \multirow[t]{4}{*}{ and visuospatial skills } & Symbol-Digit Modalities test & $50.5(8.3)$ & $29-70$ \\
\hline & Letter-Number Sequencing & $10.5(2.4)$ & $3-17$ \\
\hline & Judgment of Line Orientation & $53.8(4.3)$ & $40-60$ \\
\hline & Block Design & $37.3(11.0)$ & $10-60$ \\
\hline
\end{tabular}

CVLT $=$ California Verbal Learning Test, 2nd edition; SD = standard deviation. 
Table 5. Linear regression analyses for the association between serum hormone values and cognitive factors at baseline $(\mathrm{n}=148)^{\mathrm{a}}$

\begin{tabular}{|c|c|c|c|c|c|c|c|c|c|c|c|c|}
\hline \multirow[t]{2}{*}{$\begin{array}{l}\text { Hormone } \\
\text { measure }\end{array}$} & \multicolumn{3}{|c|}{$\begin{array}{l}\text { Verbal episodic } \\
\text { memory }\end{array}$} & \multicolumn{3}{|c|}{$\begin{array}{l}\text { Visual episodic } \\
\text { memory }\end{array}$} & \multicolumn{3}{|c|}{$\begin{array}{l}\text { Semantic } \\
\text { memory }\end{array}$} & \multicolumn{3}{|c|}{$\begin{array}{l}\text { Executive function - } \\
\text { visuospatial skills }\end{array}$} \\
\hline & $\beta$ & $\mathrm{SE}$ & $\mathrm{p}$ & $\beta$ & $\mathrm{SE}$ & $\mathrm{p}$ & $\beta$ & $\mathrm{SE}$ & $\mathrm{p}$ & $\beta$ & $\mathrm{SE}$ & $\mathrm{p}$ \\
\hline $\log$ estrone $^{\mathrm{b}}$ & 0.02 & 0.12 & 0.86 & -0.12 & 0.14 & 0.41 & 0.08 & 0.12 & 0.52 & -0.06 & 0.10 & 0.55 \\
\hline log total estradiol & -0.003 & 0.19 & 0.99 & -0.03 & 0.23 & 0.89 & 0.46 & 0.20 & $0.02 *$ & 0.19 & 0.16 & 0.26 \\
\hline log free estradiol & 0.01 & 0.15 & 0.94 & 0.01 & 0.19 & 0.98 & 0.36 & 0.16 & $0.03^{*}$ & 0.14 & 0.14 & 0.30 \\
\hline log total testosterone & -0.25 & 0.14 & $0.08 \dagger$ & -0.25 & 0.17 & 0.15 & -0.13 & 0.15 & 0.39 & -0.06 & 0.13 & 0.63 \\
\hline log free testosterone & -0.21 & 0.13 & 0.12 & -0.19 & 0.16 & 0.24 & -0.07 & 0.14 & 0.64 & -0.04 & 0.12 & 0.73 \\
\hline $\begin{array}{l}\log \text { (free testosterone / } \\
\text { free estradiol) }\end{array}$ & -0.27 & 0.15 & $0.06 \dagger$ & -0.23 & 0.18 & 0.18 & -0.41 & 0.15 & $0.007 *$ & -0.18 & 0.13 & 0.17 \\
\hline
\end{tabular}

\footnotetext{
${ }^{a}$ Adjusted for age, education, depressive symptoms and age at menopause.

${ }^{\mathrm{b}} 12$ women were missing estrone measurements $(\mathrm{n}=136)$.

*Probability $\mathrm{p}<0.05 ; \dagger 0.1 \leq \mathrm{p} \leq 0.05$.

$\mathrm{SE}=$ standard error.
} 
Table 6. Linear regression analyses for the association between serum hormone values and the two year change in cognitive function $(n=108)^{a}$

\begin{tabular}{|c|c|c|c|c|c|c|c|c|c|c|c|c|}
\hline \multirow[t]{2}{*}{$\begin{array}{l}\text { Hormone } \\
\text { measure }\end{array}$} & \multicolumn{3}{|c|}{$\begin{array}{l}\text { Verbal episodic } \\
\text { memory }\end{array}$} & \multicolumn{3}{|c|}{$\begin{array}{l}\text { Visual episodic } \\
\text { memory }\end{array}$} & \multicolumn{3}{|c|}{$\begin{array}{l}\text { Semantic } \\
\text { memory }\end{array}$} & \multicolumn{3}{|c|}{$\begin{array}{l}\text { Executive function - } \\
\text { visuospatial skills }\end{array}$} \\
\hline & $\beta$ & SE & $\mathrm{p}$ & $\beta$ & SE & $\mathrm{p}$ & $\beta$ & SE & $\mathrm{p}$ & $\beta$ & SE & $\mathrm{p}$ \\
\hline $\log$ estrone $^{b}$ & -0.04 & 0.11 & 0.69 & -0.01 & 0.13 & 0.92 & -0.00 & 0.13 & 0.99 & 0.03 & 0.08 & 0.68 \\
\hline log free estradiol & -0.13 & 0.15 & 0.42 & -0.18 & 0.18 & 0.33 & -0.21 & 0.18 & 0.24 & -0.14 & 0.11 & 0.21 \\
\hline log total testosterone & -0.26 & 0.14 & $0.06 \dagger$ & -0.03 & 0.17 & 0.87 & 0.26 & 0.16 & 0.11 & 0.02 & 0.10 & 0.84 \\
\hline $\log$ free testosterone & -0.27 & 0.13 & $0.04 *$ & -0.05 & 0.16 & 0.76 & 0.11 & 0.15 & 0.46 & -0.06 & 0.09 & 0.50 \\
\hline
\end{tabular}

${ }^{a}$ Adjusted for age, education, depressive symptoms, age at menopause and baseline cognitive score; longitudinal analyses involved 40 fewer women, who did not participate in follow-up cognitive testing.

${ }^{\mathrm{b}} 10$ women were missing estrone measurements $(\mathrm{n}=98)$.

* Probability $\mathrm{p}<0.05 ; \dagger 0.1 \leq \mathrm{p} \leq 0.05$

$\mathrm{SE}=$ standard error 
Supplementary Table 1. Linear regression analyses for the association between select serum hormone values and specific cognitive tests (post hoc analyses, $n=148)^{a}$

Supplementary Table 1A. Semantic memory factor

\begin{tabular}{|c|c|c|c|c|c|c|}
\hline \multirow[t]{2}{*}{ Hormone measure } & \multicolumn{3}{|c|}{ Boston Naming Test } & \multicolumn{3}{|c|}{ Category Fluency } \\
\hline & $\beta$ & SE & $\mathrm{p}$ & $\beta$ & SE & $\mathrm{p}$ \\
\hline log total estradiol & 0.72 & 0.23 & $0.002 *$ & 0.19 & 0.25 & 0.44 \\
\hline $\log$ free estradiol & 0.60 & 0.19 & $0.002 *$ & 0.11 & 0.21 & 0.60 \\
\hline $\log ($ free testosterone / free estradiol) & -0.44 & 0.18 & $0.02 *$ & -0.39 & 0.19 & $0.05 \dagger$ \\
\hline
\end{tabular}

Supplementary Table 1B. Verbal episodic memory factor

\begin{tabular}{|c|c|c|c|c|c|c|c|c|c|c|c|c|}
\hline \multirow[t]{2}{*}{$\begin{array}{l}\text { Hormone } \\
\text { measure }\end{array}$} & \multicolumn{3}{|c|}{$\begin{array}{l}\text { CVLT, } \\
\text { immediate recall }\end{array}$} & \multicolumn{3}{|c|}{$\begin{array}{l}\text { CVLT, } \\
\text { delayed recall }\end{array}$} & \multicolumn{3}{|c|}{$\begin{array}{l}\text { Unrelated word list, } \\
\text { immediate recall }\end{array}$} & & \multicolumn{2}{|c|}{$\begin{array}{l}\text { Unrelated word list, } \\
\text { delayed recall }\end{array}$} \\
\hline & $\beta$ & SE & $\mathrm{p}$ & $\beta$ & SE & $\mathrm{p}$ & $\beta$ & SE & $\mathrm{p}$ & $\beta$ & SE & $\mathrm{p}$ \\
\hline log total testosterone & -0.35 & 0.18 & $0.05 \dagger$ & -0.33 & 0.19 & $0.09 \dagger$ & -0.14 & 0.19 & 0.46 & -0.17 & 0.19 & 0.36 \\
\hline $\begin{array}{l}\log \text { (free testosterone / } \\
\text { free estradiol) }\end{array}$ & -0.29 & 0.18 & $0.10 \dagger$ & -0.42 & 0.19 & $0.03 *$ & -0.16 & 0.19 & 0.41 & -0.13 & 0.19 & 0.50 \\
\hline
\end{tabular}

${ }^{a}$ Adjusted for age, education, depressive symptoms and age at menopause.

* Probability $\mathrm{p}<0.05 ; \dagger 0.1 \leq \mathrm{p} \leq 0.05$.

CVLT $=$ California Verbal Learning Test, 2nd edition; $\mathrm{SE}=$ standard error. 


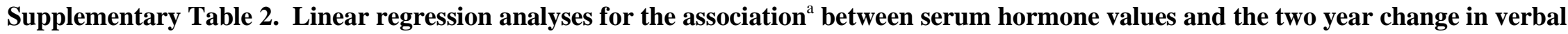
episodic memory analysis, $n=108$ )

\begin{tabular}{|c|c|c|c|c|c|c|c|c|c|c|c|c|}
\hline \multirow[t]{2}{*}{$\begin{array}{l}\text { Hormone } \\
\text { measure }\end{array}$} & \multicolumn{3}{|c|}{$\begin{array}{l}\text { CVLT, } \\
\text { immediate recall }\end{array}$} & \multicolumn{3}{|c|}{$\begin{array}{l}\text { CVLT, } \\
\text { delayed recall }\end{array}$} & \multicolumn{3}{|c|}{$\begin{array}{l}\text { Unrelated word list, } \\
\text { immediate recall }\end{array}$} & \multicolumn{3}{|c|}{$\begin{array}{l}\text { Unrelated word list, } \\
\text { delayed recall }\end{array}$} \\
\hline & $\beta$ & $\mathrm{SE}$ & $\mathrm{p}$ & $\beta$ & $\mathrm{SE}$ & $\mathrm{p}$ & $\beta$ & $\mathrm{SE}$ & $\mathrm{p}$ & $\beta$ & $\mathrm{SE}$ & $\mathrm{p}$ \\
\hline log total testosterone & -0.02 & 0.22 & 0.92 & -0.03 & 0.21 & 0.88 & -0.57 & 0.21 & $0.008 *$ & -0.59 & 0.21 & $0.005^{*}$ \\
\hline log free testosterone & -0.09 & 0.21 & 0.69 & -0.05 & 0.20 & 0.81 & -0.50 & 0.20 & $0.01 *$ & -0.60 & 0.19 & $0.003 *$ \\
\hline
\end{tabular}

${ }^{a}$ Adjusted for age, education, depressive symptoms, age at menopause and baseline cognitive score.

* Probability $\mathrm{p}<0.05$.

CVLT $=$ California Verbal Learning Test, 2nd edition; SE $=$ standard error. 